\title{
Review Article \\ The Role of SGLT2 Inhibitor on the Treatment of Diabetic Retinopathy
}

\author{
Wenjun Sha, ${ }^{1}$ Song Wen, ${ }^{2}$ Lin Chen, ${ }^{1}$ Bilin Xu, ${ }^{1}$ Tao Lei $\mathbb{D}^{1},{ }^{1}$ and Ligang Zhou $\mathbb{D}^{2}$ \\ ${ }^{1}$ Department of Endocrinology and Metabolism, Putuo Hospital, Shanghai University of Traditional Chinese Medicine, \\ Shanghai 200062, China \\ ${ }^{2}$ Department of Endocrinology, Shanghai Pudong Hospital, Fudan University, Shanghai 201399, China
}

Correspondence should be addressed to Tao Lei; leitao5899@126.com and Ligang Zhou; zhouligang@yahoo.com

Received 6 August 2020; Revised 16 October 2020; Accepted 23 October 2020; Published 12 November 2020

Academic Editor: Dominika Pohlmann

Copyright (c) 2020 Wenjun Sha et al. This is an open access article distributed under the Creative Commons Attribution License, which permits unrestricted use, distribution, and reproduction in any medium, provided the original work is properly cited.

Diabetic retinopathy (DR) is one of the most serious complications of diabetic microangiopathy. DR has an early onset and is not easy to detect. When visual impairment occurs, the optimal period for therapy is often missed. Therefore, the prevention and treatment of DR should start from the early stage of diabetes. Sodium-dependent glucose transporter 2 inhibitor (SGLT2i) is a new antidiabetic drug which is mainly used in clinical practice to control blood glucose of patients with type 2 diabetes prone to develop chronic heart failure. Recent studies have found that SGLT2 is also expressed in the human retina. Now, the prevention and treatment of diabetic retinopathy with SGLT2i while reducing blood sugar has become a new research field. Hence, this article reviewed the recent therapeutic and research progress of SGLT2 in the treatment of diabetic retinopathy.

\section{Introduction}

Diabetes is a group of metabolic diseases characterized by hyperglycemia and microvascular and macrovascular complications caused by insulin secretion defect and/or its biological function disorder [1-3]. According to the International Diabetes Federation (IDF), there were 425 million people with diabetes aged 20-79 worldwide in 2017, and the number will increase to 629 million at the middle of this century [4]. Diabetic retinopathy (DR) is one of the most common and serious microvascular complications in diabetes. Studies demonstrate that the incidence of DR increases with the course of diabetes.

An epidemiological survey in 2011 showed that the incidence of DR is about $25 \%$ of 5 years after the diagnosis of diabetes, $60 \%$ after 10 years of it, and up to $75 \% \sim 80 \%$ after 15 years of it [5]. At the same time, DR is also the main cause of visual impairment and irreversible blindness in middleaged and elderly people. According to the data published by the World Health Organization in 2002, DR worldwide caused about $4.8 \%$ of the 37 million cases of blindness [6].

\section{The Classification of Diabetic Retinopathy}

The classification of DR development is related to the abnormalities of retinal microvascular system, including increased blood retinal barrier permeability, decreased vascular endothelial cells and pericytes, thickened vascular basement membrane, capillary occlusion, retinal neurons, and glial abnormalities. Therefore, DR is currently divided into nonproliferative diabetic retinopathy (NPDR) and proliferative diabetic retinopathy (PDR).

2.1. Nonproliferative Diabetic Retinopathy (NPDR). Nonproliferative diabetic retinopathy (NPDR) occurs in the early stage of DR. The main pathophysiological basis is high glucose-induced retinal degeneration, including loss of capillary pericytes, thickening of the basement membrane, thinning of the blood vessel layer and destruction of the blood-retinal barrier, changes in the broken line leading to retinal hemorrhage and arteriole tumors, and microvascular abnormalities in the retina and cotton wool spots (fluffy white plaques on the retina caused by local swelling of the 
retinal nerve fiber layer) [7]. However, in the NPDR stage, patients are usually asymptomatic and have normal vision. When visual impairment occurs, the best period of treatment is often missed [8]. Therefore, it is necessary to prevent the occurrence of DR in the early stage of diabetes.

2.2. Proliferative Diabetic Retinopathy (PDR). Studies have found that the prevalence of PDR is close to $50 \%$ after 25 years of diabetes diagnosis, and most patients with type 1 diabetes (T1DM) will develop PDR after about 10 years [9]. At the PDR stage, the new blood vessels in the eye are continuously generated and often accompanied by the oozing and hyperplasia of the eye tissue, which can destroy the normal structure and function of the eye and ultimately lead to the visual impairment of the patient [10]. Compared with NPDR, PDR is more harmful to eyesight and could cause severe vision loss or even complete blindness.

\section{The Mechanism for Diabetic Retinopathy}

The pathogenesis of DR is complex, and the mechanism has not yet been fully elucidated. The pathological mechanism hypotheses currently proposed mainly include the theory of chronic inflammation, retinal hemodynamic changes, oxidative stress, gene polymorphism, and neurodegenerative changes (Figure 1).

3.1. Inflammation. Chronic hyperglycemia leads to oxidative damage of retinal capillary wall and vascular occlusion by activating various metabolic pathways. In the early stage of hyperglycemia, transcription factors are activated to elevate proinflammatory factors, triggering the activation of microglia cells and resulting in low-grade inflammation on the retina [11]. Secondly, the increase of icAM-1 expression in retinal endothelial cells and the accumulation of white blood cells around the retinal capillary walls will cause the retina to produce low-grade inflammation through the release of cytokines, chemokines, proinflammatory factors, and proangiogenic growth factors [12].

This low-grade inflammation destroys the tight junctions between endothelial cells by pericyte injury [13], increasing the permeability of blood vessels, exuding serum and weakening the vascular wall barrier, destroying the retinal blood barrier (BRB), and causing edema in the retina. Because of edema, the extracellular hydrostatic pressure increases, causing the capillary network to collapse and increase occlusion, leading to increased retinal ischemia, glycosylation, thickening of the endothelial basement membrane, development of vascular malformations, and formation of microaneurysms [11].

3.2. Oxidative Stress and Free Radicals. There are a large number of polyunsaturated fatty acids on the retina, which makes it particularly sensitive to oxidative stress [14]. In the process of glucose oxidation, nonenzymatic saccharification of proteins occurs and reactive oxygen species (ROS) is produced. Hyperglycemia leads to an increase in the synthesis of ROS in the retina, which acts as a coinitiating factor to activate four classical molecular pathways [15], namely, abnormal polyol pathway, inositol metabolism pathway, accumulation of protein nonenzymatic glycosylation end

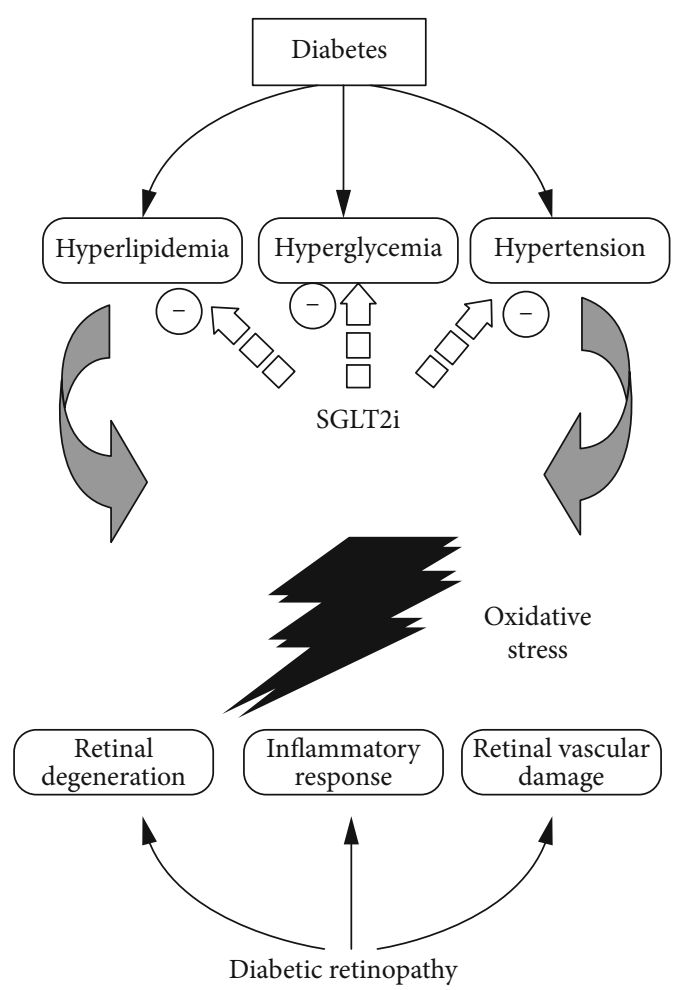

FIGURE 1: The proposed mechanism of SGLT2 inhibitor (SGLT2i) against diabetic retinopathy, in which SGLT2i improves hyperlipidemia, hyperglycemia, and hypertension.

products [16], and protein kinase C (PKC) activates [17] and acts on the angiotensin-converting enzyme system [18]. The activation of each pathway further promotes the generation of ROS with positive feedback, which enhances oxidation and damages the blood-retinal barrier structure, which ultimately leads to the occurrence of DR.

\section{The Treatment of New Diabetic Drug SGLT2 Inhibitor on the DR Treatment}

In 1835, Petersen extracted Phlorizin, the nonselective sodium-dependent glucose transporters (SGLT) inhibitor from apple roots [19]. In further animal models, it was found to increase urine glucose excretion and reduce hyperglycemia. However, Phlorizin is prone to cause adverse reactions of the digestive tract and has low utilization efficiency, so it is not used in the treatment of diabetes [20]. Modern studies have found that SGLT2 belongs to the superfamily of sodium-glucose cotransporters. It is a low-affinity, highefficiency transporter. It is not only distributed in the proximal convoluted tubules S1 and S2 in the kidney but also in the lens and the retina [21]. SGLT2 provides protection for the subtle nutrient metabolism of the eye [22]. Based on this, sodium-glucose transport synergistic protein 2 inhibitor (SGLT2i) is independent of hypoglycemic effect and also has DR protective effect.

4.1. Improvement of Factors of Diabetic Pathogenesis. There are many risk factors affecting the occurrence of DR, 
including poor blood sugar control, hypertension, and hyperlipidemia. Improving these influence factors can effectively delay the progress of DR [23].

4.1.1. Control of Hyperglycemia. It is known that the microvascular complications of type 2 diabetes (T2DM) are related to hyperglycemia. UKPDS has shown that a $1 \%$ reduction in HbAlc can reduce the risk of microvascular complications by $37 \%$ [24]. For every $1 \mathrm{mmol} / \mathrm{L}$ decrease in blood glucose level, the risk of DR will decrease by $21 \%$ [25]. In the "Diagnostic Standards for Diabetes Medicine" issued by the American Diabetes Association (ADA) in 2020, the new hypoglycemic drug SGLT2i is recommended as a first-line drug in the patients with chronic heart failure. In the first week of treatment, it can reduce fasting blood glucose by about $1.5 \mathrm{mmol} / \mathrm{L}$ and effectively reduce $\mathrm{HbAlc}$ by about $1.5 \%$, which is equivalent to the efficacy of metformin $2000 \mathrm{mg}$ per day. Due to its multiple benefits in addition to hypoglycemic effect, SGLT2i could also be administrated in appropriate diabetic patients [26].

4.1.2. Control of Hypertension. In T2DM patients with hypertension, strict control of blood pressure can delay the progression of DR and the deterioration of visual acuity [27]. Studies have confirmed that for every $10 \mathrm{mmHg}$ drop in systolic blood pressure (SBP), the risk of diabetic microvascular complications can be reduced by $13 \%$, and strict blood pressure control can reduce the risk of blindness in DR by $47 \%$ [28]. Elevated 24-hour SBP is independently associated with higher wall-to-lumen ratio (WLR) in retinal arterioles [29]. A clinical study involving 311 centers in 16 countries has confirmed that SGLT2i has a significant antihypertensive effect, which can reduce SBP by $11.9 \mathrm{mmHg}$ on average. SGLT2i combined with other common hypertension drugs can achieve more satisfactory antihypertensive effect [30]. At present, some scholars believe that the antihypertensive mechanism of SGLT2i should be related to the osmotic diuresis caused by glucose excretion [31]. Some other scholars also believe that its antihypertensive effect is related to the inhibitory effect on the renin-angiotensin-aldosterone system when promoting urine excretion. Dapagliflozin improves muscle insulin sensitivity but enhances endogenous glucose production [32,33]. Clinical studies found that BP parameters were lower than baseline 6 weeks after Dagliazine treatment. Therefore, Dagliazine may prevent retinal wall remodeling by lowering BP [34].

4.1.3. Control of Hyperlipidemia. Dyslipidemia also promotes the development of DR. The hard exudation of DR is the exudate of lipid and protein that occurs in the plexiform layer of the retina. It has been found that the severity of hard exudation of DR is positively correlated with triglyceride (TG), low-density lipoprotein (LDL), and total cholesterol (TC) levels and negatively correlated with high-density lipoprotein (HDL) levels [35]. Blood lipid control can improve the function of retinal vascular endothelial cells, reduce inflammation and leakage of fundus microvessels, and thus delay the development of DR [36]. The effect of SGLT2i on blood lipids is multifold. It can be observed that total cholesterol increa- se/no change, triacylglycerol decrease/no change, and LDL$\mathrm{C}$ and high-density lipoprotein cholesterol (HDL-C) increase by $5 \% \sim 10 \%$, but LDL-C/HDL-C ratio is not affected [37]. In addition, small and dense LDL-C was reduced by $20 \% \sim 30 \%$ after SGLT2i treatment [38].

4.2. Protection of Blood-Retinal Barrier. DR is characterized by the destruction of the blood-retinal barrier (BRB) and leakage of retinal vascular. Normal retina has two layers, an inner and outer BRB. There are two types of BRBs in the normal retina. The inner barrier is composed of zonula occludens between retinal capillary endothelial cells and pericytes; and the outer barrier is composed of retinal pigment epithelium (RPE) layer and the small closed zone between them. Hyperglycemia, ischemia, hypoxia, oxidative stress, inflammation, and inflammatory factors are the main predisposing factors for the destruction of BRB caused by diabetes [39]. The destruction of the inner BRB caused by DR is the main cause of vascular leakage.

SGLT2 acts as a glucose sensor in the retinal microvasculature. Excessive entry of $\mathrm{Na}^{+}$-dependent glucose can cause intracellular swelling of pericytes [40] and lead to loss of contractile function, death of pericytes, and overperfusion of the retina. The overexpression of the extracellular matrix (such as fibronectin, collagen IV, and laminin) is associated with the thickening of the basement membrane, subsequent microvascular occlusion, and insufficient retinal perfusion [41]. This change in retinal hemodynamics can lead to multiple downstream triggers of DR. SGLT2 inhibitors have been shown to reduce pericyte swelling and extracellular matrix overexpression [42]. Early suppression of hemodynamic changes in DR is of great significance for the development of effective DR treatments [43].

Takakura and others have shown the effect of iplegliflozin on the diabetic retina of spontaneously diabetic Torii obese rats. In this study, diabetic rats treated with iplegliflozin showed a decrease in the oscillating potential on the electroretinogram, the outer nuclear layer of the neural retina was irregular, and iplegliflozin inhibited the progression of lens cataract formation [44].

4.3. Protection of the Fundus Capillaries. Arteriole remodeling is a factor leading to the progression of diabetic retinopathy [45]. In PDR, the basement membrane of retinal capillaries thickens, pericytes gradually disappear, microangiomas form, endothelial cells proliferate, luminal occlusion, and finally new blood vessels form. Many increases in WLR and cross-sectional area are characteristic of vascular hypertrophy [46]. Multiple clinical and animal studies have shown that the occurrence and development of DR are related to the increase of WLR and crosssectional area of arteriolar walls. Grunwald et al. found that in patients with type 1 diabetes and background retinopathy with poor blood glucose control, retinal total volume blood flow was $23 \%$ higher than normal [47]. Patel et al. found that compared with patients without diabetes, diabetic patients with signs of diabetic retinopathy had higher blood flow and larger blood vessel diameter [48]. This increased blood flow may cause vascular damage by 
increasing shear stress, leading to endothelial dysfunction, basement membrane disruption, and extracellular matrix remodeling [49].

A prospective, single-center, placebo-controlled, doubleblind, randomised crossover phase IIIb study evaluated the effects of dapagliflozin on the retinal microvasculature, showing that compared with the placebo group, dagrazine treatment group did not increase the retinal wall-cavity ratio and reduced retinal capillary flow, suggesting that dagrazine could prevent vascular hyperemia [50]. In addition, the treatment seemed to prevent structural changes in retinal arterioles. This may be due in part to the drug's ability to reduce glucose resulting in reduced blood flow, reduced subcutaneous fibrin deposition, altered smooth muscle cells, and prevented arteriolar wall thickening [51].

4.4. Protection of the Optic Nerve. The optic nerve is a special somatosensory nerve, which is very sensitive to ischemia and hypoxia. The optic nerve is made up of ganglion cell axons. The surface of its nerve fibers has only myelin sheath and no nerve membrane. Once it undergoes apoptosis, it is difficult to regenerate. The progressive apoptosis of optic nerve cells is the main cause of irreversible damage to visual function. There are roughly three most important mechanisms for neurodegeneration in patients with DR: accumulation of extracellular glutamate, oxidative stress, and reduction of neuroprotective factors synthesized by the retina [52].

Recent findings indicate that there is a correlation between SGLT2 expression and the sympathetic nervous system. Hyperfunction of the sympathetic nervous system (SNS) is a feature of obesity and T2DM [53]. We showed for the first time that norepinephrine, the main neurotransmitter of SNS, upregulates the expression of SGLT2 protein in human proximal renal tubular cells [54]. In addition, high-fat diet-fed mice treated with dapagliflozin showed decreased tissue norepinephrine content and tyrosine hydroxylase expression in both heart and kidney tissues, indicating SGLT2 inhibitors in our animal model. It has significant sympathetic inhibition [55]. So far, limited studies have studied the autonomic nervous system associated with $\mathrm{DR}$, and these studies have shown that DR is associated with early autonomic dysfunction in patients with T1D and T2D [56]. The balance between sympathetic and parasympathetic effects determines the normal function of the autonomic nervous system. We have generated new data highlighting that in a mouse model of neuropathic hypertension with substantially activated SNS, nerve damage in the outer layer of the retina is obvious, which is DR. Therefore, SGLT2i may also reduce harmful retinal changes that may be supported by SNS overactivation.

To sum up, SGLT2i has been widely used in the clinical treatment of diabetes. However, the data on their efficacy in diabetic complications, especially in the treatment of DR, were mainly derived from animal experiments. These animal experimental data and conclusions allow us to see the great clinical application potential of SGLT2i in the treatment of DR. It is believed that with more clinical studies on SGLT2i, clinical data on the efficacy of DR will be more abundant.

\section{Conflicts of Interest}

The authors declare no conflict of interest.

\section{Authors' Contributions}

Wenjun Sha and Song Wen contributed equally to this work.

\section{Acknowledgments}

This work was supported by the National Natural Science Foundation of China (81774083, 81704027, and 81370932), Key Subject Construction Project of Shanghai Municipal Health and Family Planning Commission (ZK2019B16), Special Department Fund of the Pudong New Area Health Planning Commission (WZzk2017-03), Integrative Medicine Special Fund of Shanghai Municipal Health Planning Committee (ZHYY-ZXYJHZX-2-201712), Shanghai Municipal Health and Family Planning Commission (201840345), Outstanding Leaders Training Program of Pudong Health Bureau of Shanghai (PWR12014-06), Outstanding Clinical Discipline Project of Shanghai Pudong (PWYgy-2018-08), Natural Science Foundation of China (21675034), and Shanghai Natural Science Foundation (19ZR1447500).

\section{References}

[1] American Diabetes Association, "2. Classification and diagnosis of diabetes: standards of medical care in diabetes-2019," Diabetes Care, vol. 42, Supplement 1, pp. S13-s28, 2019.

[2] M. Gong, S. Wen, T. Nguyen, C. Wang, J. Jin, and L. Zhou, "Converging relationships of obesity and hyperuricemia with special reference to metabolic disorders and plausible therapeutic implications," Diabetes, Metabolic Syndrome and Obesity: Targets and Therapy, vol. 13, pp. 943-962, 2020.

[3] S. Wen, C. Wang, M. Gong, and L. Zhou, "An overview of energy and metabolic regulation," Science China Life Sciences, vol. 62, no. 6, pp. 771-790, 2019.

[4] T. Y. Wong and C. Sabanayagam, "The war on diabetic retinopathy," Asia-Pacific Journal of Ophthalmology, vol. 8, no. 6, pp. 448-456, 2019.

[5] G. P. Giuliari, "Diabetic retinopathy: current and new treatment options," Current Diabetes Reviews, vol. 8, no. 1, pp. 32-41, 2012.

[6] D. S. W. Ting, G. C. M. Cheung, and T. Y. Wong, "Diabetic retinopathy: global prevalence, major risk factors, screening practices and public health challenges: a review," Clinical \& Experimental Ophthalmology, vol. 44, no. 4, pp. 260-277, 2016.

[7] R. P. Homme, M. Singh, A. Majumder et al., "Remodeling of retinal architecture in diabetic retinopathy: disruption of ocular physiology and visual functions by inflammatory gene products and pyroptosis," Frontiers in Physiology, vol. 9, p. $1268,2018$.

[8] P. H. Scanlon, S. J. Aldington, and I. M. Stratton, "Epidemiological issues in diabetic retinopathy," Middle East African Journal of Ophthalmology, vol. 20, no. 4, pp. 293-300, 2013.

[9] D. P. Hainsworth, I. Bebu, L. P. Aiello et al., "Risk factors for retinopathy in type 1 diabetes: the DCCT/EDIC study," Diabetes Care, vol. 42, no. 5, pp. 875-882, 2019.

[10] K. Kaku, S. Inoue, O. Matsuoka et al., "Efficacy and safety of dapagliflozin as a monotherapy for type 2 diabetes mellitus 
in Japanese patients with inadequate glycaemic control: a phase II multicentre, randomized, double-blind, placebocontrolled trial," Diabetes, Obesity \& Metabolism, vol. 15, no. 5, pp. 432-440, 2013.

[11] F. Semeraro, F. Morescalchi, A. Cancarini, A. Russo, S. Rezzola, and C. Costagliola, "Diabetic retinopathy, a vascular and inflammatory disease: therapeutic implications," Diabetes \& Metabolism, vol. 45, no. 6, pp. 517-527, 2019.

[12] M. Mesquida, F. Drawnel, and S. Fauser, "The role of inflammation in diabetic eye disease," Seminars in Immunopathology, vol. 41, no. 4, pp. 427-445, 2019.

[13] B. G. Spencer, J. J. Estevez, E. Liu, J. E. Craig, and J. W. Finnie, "Pericytes, inflammation, and diabetic retinopathy," Inflammopharmacology, vol. 28, no. 3, pp. 697-709, 2020.

[14] O. M. Ighodaro, "Molecular pathways associated with oxidative stress in diabetes mellitus," Biomedicine \& Pharmacother$a p y$, vol. 108, pp. 656-662, 2018.

[15] M. Benlarbi-Ben Khedher, K. Hajri, A. Dellaa et al., "Astaxanthin inhibits aldose reductase activity in Psammomys obesus, a model of type 2 diabetes and diabetic retinopathy," Food Science \& Nutrition, vol. 7, no. 12, pp. 3979-3985, 2019.

[16] M. Mishra, A. J. Duraisamy, S. Bhattacharjee, and R. A. Kowluru, "Adaptor protein p66Shc: a link between cytosolic and mitochondrial dysfunction in the development of diabetic retinopathy," Antioxidants \& Redox Signaling, vol. 30, no. 13, pp. 1621-1634, 2019.

[17] H. L. Deissler and G. E. Lang, "The protein kinase C inhibitor: ruboxistaurin," Developments in Ophthalmology, vol. 55, pp. 295-301, 2016.

[18] S. Luo, C. Shi, F. Wang, and Z. Wu, "Association between the angiotensin-converting enzyme (ACE) genetic polymorphism and diabetic retinopathy-a meta-analysis comprising 10,168 subjects," International Journal of Environmental Research and Public Health, vol. 13, no. 11, article 1142, 2016.

[19] J. R. L. Ehrenkranz, N. G. Lewis, C. Ronald Kahn, and J. Roth, "Phlorizin: a review," Diabetes/Metabolism Research and Reviews, vol. 21, no. 1, pp. 31-38, 2005.

[20] M. Uldry and B. Thorens, "The SLC2 family of facilitated hexose and polyol transporters," Pflügers Archiv, vol. 447, no. 5, pp. 480-489, 2004.

[21] T. Yakovleva, V. Sokolov, L. Chu et al., "Comparison of the urinary glucose excretion contributions of SGLT2 and SGLT1: a quantitative systems pharmacology analysis in healthy individuals and patients with type 2 diabetes treated with SGLT2 inhibitors," Diabetes, Obesity \& Metabolism, vol. 21, no. 12, pp. 2684-2693, 2019.

[22] M. May, T. Framke, B. Junker, C. Framme, A. Pielen, and C. Schindler, "How and why SGLT2 inhibitors should be explored as potential treatment option in diabetic retinopathy: clinical concept and methodology," Therapeutic Advances in Endocrinology and Metabolism, vol. 10, article 2042018819891886, 2019.

[23] J. Dziuba, P. Alperin, J. Racketa et al., "Modeling effects of SGLT-2 inhibitor dapagliflozin treatment versus standard diabetes therapy on cardiovascular and microvascular outcomes," Diabetes, Obesity \& Metabolism, vol. 16, no. 7, pp. 628-635, 2014.

[24] I. M. Stratton, A. I. Adler, H. A. Neil et al., "Association of glycaemia with macrovascular and microvascular complications of type 2 diabetes (UKPDS 35): prospective observational study," BMJ, vol. 321, no. 7258, pp. 405-412, 2000.
[25] N. K. Wang, C. C. Lai, J. P. Wang et al., "Risk factors associated with the development of retinopathy $10 \mathrm{yr}$ after the diagnosis of juvenile-onset type 1 diabetes in Taiwan: a cohort study from the CGJDES," Pediatric Diabetes, vol. 17, no. 6, pp. 407-416, 2016.

[26] American Diabetes Association, "1. Improving care and promoting health in populations: standards of medical care in diabetes-2020," Diabetes Care, vol. 43, Supplement 1, pp. S7-s13, 2020.

[27] UK Prospective Diabetes Study Group, “Tight blood pressure control and risk of macrovascular and microvascular complications in type 2 diabetes: UKPDS 38," BMJ, vol. 317, no. 7160, pp. 703-713, 1998.

[28] S. Wang, L. Xu, J. B. Jonas et al., "Major eye diseases and risk factors associated with systemic hypertension in an adult Chinese population," Ophthalmology, vol. 116, no. 12, pp. 2373-2380, 2009.

[29] M. Salvetti, C. Agabiti Rosei, A. Paini et al., "Relationship of wall-to-lumen ratio of retinal arterioles with clinic and 24hour blood pressure," Hypertension, vol. 63, no. 5, pp. 11101115, 2014.

[30] A. Merovci, C. Solis-Herrera, G. Daniele et al., "Dapagliflozin improves muscle insulin sensitivity but enhances endogenous glucose production," The Journal of Clinical Investigation, vol. 124, no. 2, pp. 509-514, 2014.

[31] B. Peene and K. Benhalima, "Sodium glucose transporter protein 2 inhibitors: focusing on the kidney to treat type 2 diabetes," Therapeutic Advances in Endocrinology and Metabolism, vol. 5, no. 5, pp. 124-136, 2014.

[32] S. J. Shin, S. Chung, S. J. Kim et al., "Effect of sodium-glucose co-transporter 2 inhibitor, dapagliflozin, on renal reninangiotensin system in an animal model of type 2 diabetes," PLoS One, vol. 11, no. 11, article e0165703, 2016.

[33] B. Wang, F. Wang, Y. Zhang et al., "Effects of RAS inhibitors on diabetic retinopathy: a systematic review and meta-analysis," The Lancet Diabetes and Endocrinology, vol. 3, no. 4, pp. 263-274, 2015.

[34] E. G. Dorsey-Treviño, B. M. Contreras-Garza, J. G. GonzálezGonzález et al., "Systematic review and meta-analysis of the effect of SGLT-2 inhibitors on microvascular outcomes in patients with type 2 diabetes: a review protocol," BMJ Open, vol. 8, no. 6, article e020692, 2018.

[35] H. Y. Zhang, J. Y. Wang, G. S. Ying, L. P. Shen, and Z. Zhang, "Serum lipids and other risk factors for diabetic retinopathy in Chinese type 2 diabetic patients," Journal of Zhejiang University Science B, vol. 14, no. 5, pp. 392-399, 2013.

[36] J. Li, J. J. Wang, D. Chen et al., "Systemic administration of HMG-CoA reductase inhibitor protects the blood-retinal barrier and ameliorates retinal inflammation in type 2 diabetes," Experimental Eye Research, vol. 89, no. 1, pp. 71-78, 2009.

[37] S. Filippas-Ntekouan, V. Tsimihodimos, T. Filippatos, T. Dimitriou, and M. Elisaf, "SGLT-2 inhibitors: pharmacokinetics characteristics and effects on lipids," Expert Opinion on Drug Metabolism \& Toxicology, vol. 14, no. 11, pp. 1113-1121, 2018.

[38] E. Ferrannini, "Sodium-glucose co-transporters and their inhibition: clinical physiology," Cell Metabolism, vol. 26, no. 1, pp. 27-38, 2017.

[39] D. H. Jo, J. H. Yun, C. S. Cho, J. H. Kim, J. H. Kim, and C. H. Cho, "Interaction between microglia and retinal pigment epithelial cells determines the integrity of outer blood-retinal 
barrier in diabetic retinopathy," Glia, vol. 67, no. 2, pp. 321$331,2019$.

[40] M. Wakisaka, M. Yoshinari, S. Nakamura et al., "Suppression of sodium-dependent glucose uptake by captopril improves high-glucose-induced morphological and functional changes of cultured bovine retinal pericytes," Microvascular Research, vol. 58, no. 3, pp. 215-223, 1999.

[41] S. Roy, E. Bae, S. Amin, and D. Kim, "Extracellular matrix, gap junctions, and retinal vascular homeostasis in diabetic retinopathy," Experimental Eye Research, vol. 133, pp. 58-68, 2015.

[42] H. Yoshizumi, T. Ejima, T. Nagao, and M. Wakisaka, "Recovery from diabetic macular edema in a diabetic patient after minimal dose of a sodium glucose co-transporter 2 inhibitor," Am J Case Rep, vol. 19, pp. 462-466, 2018.

[43] Y. Y. Chen, T. T. Wu, C. Y. Ho et al., "Dapagliflozin prevents NOX- and SGLT2-dependent oxidative stress in lens cells exposed to fructose-induced diabetes mellitus," International Journal of Molecular Sciences, vol. 20, no. 18, article 4357, 2019.

[44] S. Takakura, T. Toyoshi, Y. Hayashizaki, and T. Takasu, "Effect of ipragliflozin, an SGLT2 inhibitor, on progression of diabetic microvascular complications in spontaneously diabetic Torii fatty rats," Life Sciences, vol. 147, pp. 125-131, 2016.

[45] C. Ott, A. Jumar, K. Striepe et al., "A randomised study of the impact of the SGLT2 inhibitor dapagliflozin on microvascular and macrovascular circulation," Cardiovascular Diabetology, vol. 16, no. 1, p. 26, 2017.

[46] A. Jumar, C. Ott, I. Kistner et al., "Early signs of end-organ damage in retinal arterioles in patients with type 2 diabetes compared to hypertensive patients," Microcirculation, vol. 23, no. 6, pp. 447-455, 2016.

[47] J. E. Grunwald, J. DuPont, and C. E. Riva, "Retinal haemodynamics in patients with early diabetes mellitus," The British Journal of Ophthalmology, vol. 80, no. 4, pp. 327-331, 1996.

[48] V. Patel, S. Rassam, R. Newsom, J. Wiek, and E. Kohner, "Retinal blood flow in diabetic retinopathy," BMJ, vol. 305, no. 6855, pp. 678-683, 1992.

[49] O. Simó-Servat, R. Simó, and C. Hernández, "Circulating biomarkers of diabetic retinopathy: an overview based on physiopathology," Journal Diabetes Research, vol. 2016, article 5263798, pp. 1-13, 2016.

[50] J. Green, A. Yurdagul Jr., M. C. McInnis, P. Albert, and A. W. Orr, "Flow patterns regulate hyperglycemia-induced subendothelial matrix remodeling during early atherogenesis," Atherosclerosis, vol. 232, no. 2, pp. 277-284, 2014.

[51] P. Lacolley, V. Regnault, A. Nicoletti, Z. Li, and J. B. Michel, "The vascular smooth muscle cell in arterial pathology: a cell that can take on multiple roles," Cardiovascular Research, vol. 95, no. 2, pp. 194-204, 2012.

[52] R. Simó, C. Hernández, and C. Hernández, "Neurodegeneration in the diabetic eye: new insights and therapeutic perspectives," Trends in Endocrinology and Metabolism, vol. 25, no. 1, pp. 23-33, 2014.

[53] N. E. Straznicky, M. T. Grima, N. Eikelis et al., "The effects of weight loss versus weight loss maintenance on sympathetic nervous system activity and metabolic syndrome components," The Journal of Clinical Endocrinology and Metabolism, vol. 96, no. 3, pp. E503-E508, 2011.

[54] A. A. Thorp and M. P. Schlaich, "Relevance of sympathetic nervous system activation in obesity and metabolic syn- drome," Journal Diabetes Research, vol. 2015, article 341583, pp. 1-11, 2015.

[55] V. B. Matthews, R. H. Elliot, C. Rudnicka, J. Hricova, L. Herat, and M. P. Schlaich, "Role of the sympathetic nervous system in regulation of the sodium glucose cotransporter 2," Journal of Hypertension, vol. 35, no. 10, pp. 2059-2068, 2017.

[56] L. Y. Herat, V. B. Matthews, P. E. Rakoczy, R. Carnagarin, and M. Schlaich, "Focusing on sodium glucose cotransporter-2 and the sympathetic nervous system: potential impact in diabetic retinopathy," International Journal of Endocrinology, vol. 2018, Article ID 9254126, 8 pages, 2018. 